\title{
EXPRESSÃO CITOFOTOMÉTRICA DOS MARCADORES TUMORAIS KI- 67 E CD34 NO ADENOCARCINOMA DE ESTÔMAGO
}

\section{Citophotometric expression of tumoral markers: Ki67 and CD34 in stomach adenocarcinoma}

\author{
José Augusto Menezes Freitas de CAMPOS ${ }^{2}$; Ronaldo Mafia CUENCA ${ }^{1}$; Patrícia Werlang SCHORN ${ }^{1}$, \\ Adriano Guimarães IBIAPINA ${ }^{1}$, Glauco Antonio Bezerra JAPIASSU ${ }^{2}$, Olímpia Alves Teixeira LIMA ${ }^{1}$, \\ Paulo Henrique Freitas Farias da SILVA ${ }^{2}$, Tereza Cristina Santos CAVALCANTI ${ }^{1}$
}

Campos JAMF, Cuenca RM. Schorn PW, Ibiapina AG, Japiassu GAB, Lima OAT, Silva PHF, Cavalcanti TCS. Expressão citofotométrica dos marcadores tumorais Ki-67 e CD34 no adenocarcinoma de estômago. ABCD Arq Bras Cir Dig 2007;20(3):179-83.

RESUMO - Racional - O câncer de estômago ocupa o terceiro lugar das neoplasias malignas em todo o mundo e é a segunda causa de morte por câncer. No entanto, sua incidência está em declínio nos últimos anos, principalmente em paises como Estados Unidos, Inglaterra e Austrália, assim como onde a sua incidência sempre foi alta - Japão, China, Coréia e Leste da Ásia -, com estimativa de 780 casos anuais por 100 mil habitantes. Objetivos - Descrição da expressão citofotométrica dos marcadores Ki-67 e CD34 e comparação entre a expressão deles no adenocarcinoma de estômago. Métodos - Foram selecionados inicialmente 60 blocos com espécime de adenocarcinoma gástrico coletados nos serviços de patologia do Hospital do Gama - Brasília, DF e Hospital Dom Orione - Araguaina,TO. A imunoistoquímica com os marcadores Ki-67 e CD34 foi feita no Laboratório de Citologia e Histopalogia Ltda - CITOLAB, Curitiba, PR. Foram aproveitados do total 35 blocos para o estudo histológico e imunoistoquímico que foram analisados pelo sistema de citometria de imagem computarizado SAMBA 4000 no Instituto de Pesquisas Médicas do Hospital Universitário Evangélico de Curitiba. Foram estudados o índice de marcagem e a densidade óptica para ambos os marcadores analisados separadamente e depois comparados. Resultados - Das 35 lâminas, 15 (42,85\%) marcaram o Ki-67; 26 (74,28\%) o CD34 e 12 lâminas (34,28\%) marcaram com os dois marcadores. Quando se comparou o índice de marcagem entre Ki-67 e CD34, observou-se que existe diferença significativa $(P<0,001)$ a favor do CD34. Quando se comparou a densidade óptica entre ambos, observou-se que existe diferença significativa $(P<0,001)$ a favor do CD34. Conclusões - A média do índice de marcagem do marcador Ki-67 foi de 36,85\%, e a da densidade óptica de 29,33 pixels. O CD34 teve como índice de marcagem 84,86\% e 49,84 pixels de média para a densidade óptica. Quando se comparou o índice de marcagem entre ambos, observou-se que existe diferença significativa a favor do CD34. Quando se comparou a densidade óptica entre ambos também observou-se que existe diferença significativa a favor do CD34.

DESCRITORES - Adenocarcinoma de estômago. Marcadores tumorais. Ki-67. CD34. Imunoistoquímica.

\section{INTRODUÇÃO}

O câncer de estômago ocupa o terceiro lugar das neoplasias malignas em todo o mundo e é a segunda causa de morte por câncer ${ }^{3}$. No entanto sua incidência está em declínio nos últimos anos, principalmente em países como Estados Unidos, Inglaterra e Austrália. Este fato também é observado onde a sua incidência sempre foi alta como Japão, China, Coréia e Leste da Ásia, com estimativa de 780 casos anuais por 100 mil habitantes. A taxa de mortalidade registrada em países da América Latina, como Costa Rica, Chile e Colômbia, ainda continua elevada. Em 2006 estimaram-se no Brasil 23.200 novos casos, sendo a segunda causa de morte por câncer entre os homens e quarta entre as mulheres ${ }^{1}$.

Entre os tipos histológicos o adenocarcinoma é o mais freqüente e representa $95 \%$ dos casos. Ele pode ser do tipo difuso ou intestinal e subtipo indiferenciado ou diferenciado. Conforme o tipo histológico e seus subtipos

Trabalho realizado na ${ }^{1}$ União Educacional do Planalto Central - UNIPLAC - Brasília, DF e ${ }^{2}$ Hospital Dom Orione, Araguaína, TO, Brasil

Endereço para correspondência: José Augusto Menezes Freitas de Campos, e-mail: jmscampos@uol.com.br poderão ser observados sua maior incidência em diferentes faixas etárias assim como a maior ou menor incidência de metástases ${ }^{15}$.

Existem evidências quanto à etiopatogenia que demonstram papel importante dos hábitos sociais da população como tabaco, álcool e hábitos alimentares. Desta forma medidas como saneamento básico e assistência à saúde, comuns nos países desenvolvidos, podem diminuir na incidência desta doença. Trabalho realizado por Brito el al. ${ }^{2}$ mostrou que alimentos com nitratos, defumados, conservados em sal, pobres em vitamina A e C, pessoas que consomem pouco vegetais e frutas, estão mais expostos ao aparecimento deste tipo de câncer. Outros fatores que aumentam o risco do aparecimento são anemia perniciosa, gastrite atrófica, metaplasia intestinal e infecção pelo $H$. pylori.

O estadiamento é etapa importante no cuidado à pessoa com câncer de estômago - assim como em todos os tipos de cânceres -, feito através de critérios padronizados, onde estratificam-se os vários estágios que esta doença pode apresentar na sua evolução, norteando a melhor opção terapêutica e melhorar o prognóstico. Mas todos os esforços devem ser direcionados para o diagnóstico precoce. Com 
isso, pode-se obter controle maior de sua evolução, já que o câncer gástrico com diagnóstico precoce tem sobrevida de $95 \%$ em 5 anos $^{10}$.

Os estudos histopatológicos retrospectivos têm papel importante no planejamento terapêutico destas neoplasias, pois tornam o estadiamento mais criterioso, oferecendo melhores condições para o estudo durante a evolução destas doenças ${ }^{4}$.

A identificação de alguns genes e seus produtos protéicos como elementos relacionados ao desenvolvimento do câncer têm levado, através da imunoistoquímica, a uma nova fase de estudos. Os grandes avanços na área de informática aprimoraram o estudo destes tumores, destacando-se a citometria de imagem ou citofotometria ${ }^{13}$. Atualmente, estudos com marcadores tumorais através de imunoistoquímica vêm trazendo novos avanços para o diagnóstico precoce e, conseqüentemente, à melhor informação sobre o prognóstico. Desta forma, este estudo pretende observar o comportamento celular através dos marcadores tumorais durante as várias fases da carcinogênese do adenocarcinoma gástrico.

Os objetivos deste estudo foram efetuar a descrição da expressão citofotométrica dos marcadores Ki-67 e CD34 e a comparação da expressão deles no adenocarcinoma de estômago.

\section{MÉTODOS}

Este estudo retrospectivo foi feito analisando inicialmente 60 blocos de parafina contendo espécies de tumores malignos de estômago de pacientes operados entre 1998 e 2006 em vários hospitais do estado de Tocantins e Distrito Federal, Brasil. Os blocos, eram provenientes do arquivo de serviços de patologia do Hospital Dom Orione em Araguaina - TO e do Hospital Regional do Gama em Brasília - DF. Após a separação dos blocos eram eles encaminhados ao Laboratório CITOLAB em Curitiba onde foram submetidos à seleção dos melhores blocos para estudo imunoistoquímico, confirmação diagnóstica e confecção das lâminas para o estudo imunoistoquímico dos marcadores tumorais Ki-67 e CD34. No final desta avaliação, restaram 55 blocos, mas apenas 35 foram considerados apropriados para a leitura. Os blocos de parafina foram resfriados e cortados em cortes histológicos de 2 a 5 micra de espessura. Para cada bloco foram confeccionadas três lâminas: uma corada pela técnica de hematoxilina-eosina e uma para cada do marcador tumoral. As fitas de parafina obtidas pela microtomia foram colocadas em banho-maria e os cortes depositados nas lâminas e levadas à estufa à $60^{\circ} \mathrm{C}$ para melhor adesão dos tecidos.

A lâmina corada pela técnica de hematoxilina-eosina seguiu os passos já conhecidos e as utilizadas para a análise imunoistoquímica passaram inicialmente por preparação prévia de acordo com o seguinte protocolo: imersão das lâminas em solução de detergente neutro a $0,5 \%$ por 30 minutos; lavagem em água corrente até a remoção do detergente por no mínimo duas horas e depois secadas em estufa; processo de silanização com imersão das lâminas em acetona por dois minutos, em silano a $4 \%$, em acetona por quatro vezes e secagem em estufa.

Após a preparação prévia das lâminas elas recebiam os cortes histológicos e preparadas para o estudo imunoistoquímico propriamente dito.

$\mathrm{Na}$ etapa seguinte, foi realizada a desparafinização das lâminas, recuperação antigênica, bloqueio da peroxidase endógena e delimitação do corte histológico com caneta hidrofóbica. A seguir, foi realizada a incubação com os respectivos anticorpos da seguinte maneira: pingaram-se gotas da solução do anticorpo e diluente para cobrir todo corte, as lâminas eram levadas para bandejas e colocadas na geladeira durante toda a noite. No dia seguinte, eram lavadas com água destilada, secadas uma a uma e colocadas em tampão PBS por 5 minutos. Em seguida, foi pipetado o $2^{\circ}$ anticorpo, denominado Biotinylated Link Universal ${ }^{\mathbb{B}}$, que faz a ligação entre o $1^{\circ}$ anticorpo (CD34 ou Ki-67) e a streptoavidina. Deixou-se incubando por 30 minutos. O passo seguinte foi pipetar uma gota do cromógeno Diaminobenzidina (DAB) Biogen (Dako Cytomation) código: K 3467 sistema substrato-cromógeno para que reagisse com a peroxidase da streptoavidina, que estava ligada à biotina e esta, por sua vez, acoplada ao anticorpo 1.

Aguardou-se até que os cortes de tecido ficassem escuros, secou-se o excesso com papel-toalha e depois colocou-se em cuba com água por 20 minutos. Foi realizado a coloração com hematoxilina, colocação da lamínula e identificação das lâminas (Figuras 1 e 2).

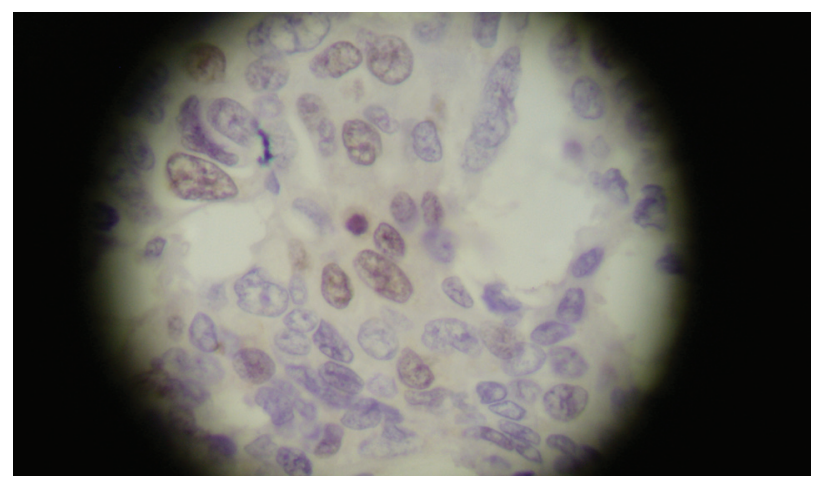

FIGURA 1 - Lâmina do adenocarcinoma de estômago marcada pelo ki-67

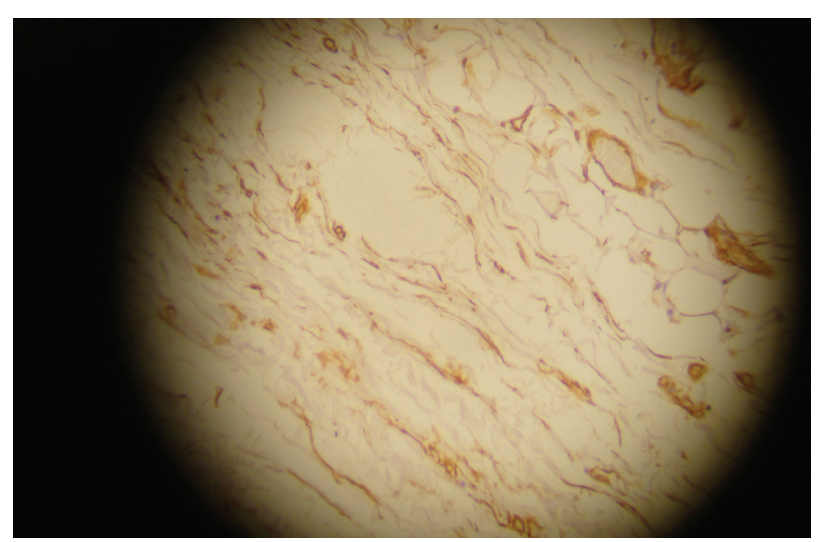

FIGURA 2 - Lâmina do adenocarcinoma de estômago marcada pelo CD34 
Terminada a confecção das lâminas foi realizada a análise quantitativa da imunoistoquímica, utilizando o "software" chamado IMMUNO ${ }^{\circledR}$ do sistema de citometria de imagem SAMBA 4000, sendo possível a leitura de 15 lâminas com marcador Ki-67 e de 26 para o marcador CD34. Foram estudados o índice de marcagem e a densidade óptica para ambos os marcadores.

A análise estatística foi realizada com o teste de ShapiroWilks, para testar a normalidade das variáveis quantitativas. A comparação dos marcadores foi feita usando-se o teste $t$ de Student para amostras pareadas.

\section{RESULTADOS}

A Tabela 1 mostra que foram lidas 15 lâminas para o marcador Ki-67 e que a média do índice de marcagem entre elas foi de $36,85 \%$, enquanto a densidade óptica apresentou média de 29,33 pixels entre as mesmas lâminas.

TABELA 1 - Índice de marcagem e densidade óptica do marcador Ki-67

\begin{tabular}{lcc}
\hline Variável & $\begin{array}{c}\text { Índice de marcagem } \\
\text { do Ki-67 }\end{array}$ & $\begin{array}{c}\text { Densidade óptica } \\
\text { do Ki-67 }\end{array}$ \\
\hline $\mathrm{n}$ & 15 & 15 \\
Média & 36,85 & 29,33 \\
Mediana & 31,92 & 26,81 \\
Mínimo & 5,43 & 12,12 \\
Maximo & 77,22 & 57,24 \\
Desvio padrão & 21,17 & 10,74 \\
\hline
\end{tabular}

O número de lâminas lidas para o marcador CD34 foram de 26 e apresentaram média do índice de marcagem de $84,86 \%$ e 49,84 pixels de média para a densidade óptica (Tabela 2).

TABELA 2 - İndice de marcagem e densidade óptica do marcador CD34

\begin{tabular}{lcc}
\hline Variável & $\begin{array}{c}\text { Índice de marcagem } \\
\text { do CD34 }\end{array}$ & $\begin{array}{c}\text { Densidade óptica } \\
\text { do CD34 }\end{array}$ \\
\hline $\mathrm{n}$ & 26 & 26 \\
Média & 84,66 & 49,84 \\
Mediana & 85,76 & 50,09 \\
Mínimo & 57,51 & 34,11 \\
Maximo & 97,56 & 66,64 \\
Desvio padrão & 9,17 & 8,48 \\
\hline
\end{tabular}

Quando se comparou o índice de marcagem entre Ki67 e CD34, cuja leitura foi coincidente em 12 lâminas, observou-se que existe diferença significante $(P<0,001)$ em favor do marcador CD34 (Tabela 3).

Quando se comparou a densidade óptica entre os marcadores Ki-67 e CD34 cuja leitura foi coincidente em 12 lâminas, observou-se que existe diferença significativa $(P<0,001)$ em favor do marcador CD34 (Tabela 4).
TABELA 3 - Comparação dos valores do índice de marcagem do Ki-67 e CD34

\begin{tabular}{lccc}
\hline Variável & $\begin{array}{c}\text { Índice de } \\
\text { marcagem } \\
\text { do CD34 }\end{array}$ & $\begin{array}{c}\text { Índice de } \\
\text { marcagem } \\
\text { do Ki-67 }\end{array}$ & $\begin{array}{c}\text { Diferença do índice de } \\
\text { marcagem (Ki-67 - CD34) }\end{array}$ \\
\hline $\mathrm{n}$ & 12 & 12 & 12 \\
Média & 83,60 & 36,55 & 47,04 \\
Mediana & 83,43 & 32,56 & 49,34 \\
Mínimo & 71,58 & 5,43 & 5,06 \\
Maximo & 97,56 & 77,22 & 76,27 \\
Desvio padrão & 8,18 & 18,94 & 23,57 \\
Valor de $P$ & & & $<\mathbf{0 , 0 0 1}$ \\
\hline
\end{tabular}

TABELA 4 - Comparação dos valores da densidade óptica do $\mathrm{Ki}-67$ e CD34

\begin{tabular}{lccc}
\hline Variável & $\begin{array}{c}\text { Densidade } \\
\text { óptica } \\
\text { do CD34 }\end{array}$ & $\begin{array}{c}\text { Densidade } \\
\text { óptica } \\
\text { do Ki-67 }\end{array}$ & $\begin{array}{c}\text { Diferença da densidade } \\
\text { óptica (Ki-67 - CD34) }\end{array}$ \\
\hline $\mathrm{n}$ & 12 & 12 & 12 \\
Média & 50,62 & 28,20 & 22,42 \\
Mediana & 50,57 & 25,89 & 21,33 \\
Mínimo & 34,13 & 12,12 & $-0,19$ \\
Maximo & 64,11 & 57,24 & 45,98 \\
Desvio padrão & 9,16 & 11,16 & 13,85 \\
Valor de $P$ & & & $<\mathbf{0 , 0 0 1}$ \\
\hline
\end{tabular}

\section{DISCUSSÃo}

No Brasil, o câncer gástrico é a segunda causa de morte por câncer entre os homens e quarta entre as mulheres. O prognóstico dos portadores de carcinoma gástrico é determinado por vários fatores, como localização, tipo histológico e tempo de evolução.

Há tendência internacional de se investir em métodos e meios de diagnóstico precoce, principalmente onde a doença tem possibilidade de cura. Pesquisadores desde a década de 90, têm realizado vários trabalhos nesta linha, com o objetivo de melhorar a sobrevida dos doentes com câncer gástrico e reconhecer epidemiologicamente os mais suscetíveis a serem acometidos por ele $e^{8,11,12,18}$.

A possibilidade de se combater o câncer abre uma nova perspectiva com o melhor entendimento da oncogênese e dos genes supressores dos tumores, permitindo-se decifrar os passos que governam a passagem de uma célula normal para cancerosa. Os estudos para detectar câncer em fase precoce têm despertado em vários pesquisadores o interesse pela biologia molecular, pois desta forma pode-se ter melhor definição do prognóstico e da conduta a ser tomada para o tratamento.

A identificação de proteínas celulares que estão envolvidas na proliferação celular e na angiogênese, tem permitido a evolução na cirurgia, na patologia e no tratamento coadjuvante do câncer gástrico. Uma série de evidências clínicas aponta para o importante papel da formação de novos vasos na formação de um tumor. Vários agentes foram inicialmente identificados como participantes na indução da neoformação vascular. $\mathrm{O}$ fator básico de crescimento 
de fibroblastos e o de crescimento vascular endotelial são apenas alguns de uma lista que inclui atualmente mais de uma dezena de agentes.

Para melhor estudar a angiogênese através dos marcadores tumorais, pode-se utilizar a citofotometria e a imunoistoquímica que identifica os marcadores relacionados ao comportamento biológico das neoplasias, com coloração das estruturas. A citometria transforma as áreas marcadas em imagens numéricas que, desta maneira, são quantificadas pelo computador.

O marcador tumoral Ki-67 marca a proliferação celular, importante fator para avaliação de câncer gástrico, bem como para orientação do tratamento e avaliação do prognóstico ${ }^{14}$. Neste estudo, o marcador Ki-67 apresentou expressão pouco significativa na análise do índice de marcagem e densidade óptica.

Para o estudo da angiogênese no processo tumoral foi utilizado o marcador CD34, descrito inicialmente por Folkman et al. 7 . Neste estudo, o CD34 apresentou resultado significativo na sua expressão para os casos estudados de carcinoma gástrico.

Contudo, entendimento maior das alterações moleculares é necessário ${ }^{6}$.

A utilização dos marcadores tumorais, da imunoistoquímica e da citofotometria encontra-se em fase de estabelecimento metodológico e reconhecimento científico no Brasil. É área nova de pesquisa que pode levar a bons esclarecimentos no processo de tumorigênese e, conse- qüentemente, ao entendimento do câncer gástrico. Suas bases são lógicas e objetivas e fazem prever grande ganho na compreensão do comportamento tumoral e sua utilização na escolha da conduta mais apropriada ao paciente.

Para a realização deste trabalho utilizou-se material de arquivo e assim como em outros estudos houve dificuldade na obtenção dos blocos de parafina e confecção das lâminas com boa qualidade, sugerindo que a aquisição a partir de material a fresco, pode ajudar na solução desta dificuldade 5 .

Os resultados encontrados neste estudo mostram que a expressão do CD34 é superior a do Ki-67 na análise do adenocarcinoma gástrico e tem coincidência com outros trabalhos na literatura ${ }^{17}$. Mas também observou-se que o Ki-67, apesar de apresentar expressão menor, tem seu valor para o diagnóstico, reafirmando a hipótese de que realmente existe uma via alternativa de proliferação nestes tumores ${ }^{9}$.

\section{CONCLUSÕES}

A média do índice de marcagem do marcador Ki-67 foi de $36,85 \%$, e a da densidade óptica de 29,33 pixels. O CD34 teve como índice de marcagem $84,86 \%$ e 49,84 pixels de média para a densidade óptica. Quando se comparou o índice de marcagem entre ambos observou-se que existe diferença significativa a favor do CD34. Quando se comparou a densidade óptica entre ambos, também se observou que existe diferença significativa a favor do CD34.

Campos JAMF, Cuenca RM. Schorn PW, Ibiapina AG, Japiassu GAB, Lima OAT, Silva PHF, Cavalcanti TCS. Citophotometric expression of tumoral markers: Ki67 and CD34 in stomach adenocarcinoma. ABCD Arq Bras Cir Dig 2007;20(3):179-83.

ABSTRACT - Background - Stomach cancer is rated as the third malignant neoplasm in the world, being the second most frequent cause of death. However, its incidence has been declining in the last few years in countries like the United States, England and Australia, and in countries where its incidence has always been high - Japan, China, Korea and in the Eastern part of Asia, which has an estimated 780 cases per year per 100,000 inhabitants. Aim - To describe the cytophotometric expression of Ki-67 and CD-34 with comparison to their expression in stomach adenocarcinomas. Methods - 60 paraffin blocks containing specimens of gastric adenocarcinomas, were initially selected in the Pathology services of the Gama Hospital - Brasília, DF an at the Dom Orione Hospital - Araguaiana, TO. The immunohistochemistry process using Ki-67 and CD-34 was performed at the Laboratório de Citologia e Histopatologia Ltda. -CITOLAB, Curitiba, PR. A total of 35 paraffin blocks were selected among the other 60 previously selected, being used in histological studies as well as for the immunohistochemistry process. These were analyzed using the computerized image cytophotometric system (SAMBA 4000) at the Institute of Medical Research of the Hospital Universitário Evangélico de Curitiba. The parameters studies and analyzed through this system were the labeling index and optical density for both of the tumoral markers being independently analyzed and latter compared with each other. Results - Of the 35 slides, 15(42,85\%) showed the expression of Ki-67; 26 (74,28\%) of CD-34 and 12 slides $(34,28 \%)$ showed the expression of both markers. When the labeling indexes between Ki-67 and CD-34 were compared, a significant difference was observed $(P<0,001)$ favoring CD-34. When the optical density of the two markers was compared, a significant difference $(P<0,001)$ was also observed, favoring CD-34. Conclusions- The average labeling index for Ki-67 was of 36,85\%, where as the optical density was of 29,33 pixels. CD-34 showed a labeling index of $84,86 \%$ and an average 49,84 pixels regarding the optical density. When the labeling index and optical density were compared between both of the markers, a significant difference was established favoring CD-34 in both of the parameters analyzed.

HEADINGS - Stomach neoplasm. Immunohistochemistry. CD-34.

\section{REFERÊNCIAS}

1. Brasil. Ministério da Saúde. Secretaria nacional de assistência à saúde. Instituto Nacional de Câncer. Estimativa da Incidência e Mortalidade por Câncer no Brasil. 2006. Rio de Janeiro: INCA, 2006.

2. Britto AV. Câncer de estômago. Cad. Saúde Pública, 13(1):1, 1997

3. Correa P. Is gastric cancer preventable? Gut, 2004;53:1217-9.

4. Cuenca MR. Estudo citofotométrico comparativo do ADN entre o carcinoma gástrico da pequena e grande curvatura do estômago. Curitiba, 2000. Tese (Doutorado em Clínica Cirúrgica - Setor de Ciências da Saúde, Universidade Federal do Paraná.

5. Danova M, Mazzini G, Wilson, G, Ucci, G, Dionigi P, Riccardi A, Fiocca R.
Ploidy and proliferative activity of human gastric carcinoma: a cytofluorometric study on fresh and on paraffin embedded material. Basic Appl Histochem, 1987; 31(1):73-82.

6. Dicken BJ, et al. Gastric adenocarcinoma: Review and considerations for future directions. Annals of Surgery, 2005;241(1):27-39.

7. Folkman J, Merler E, Abernathy C, Williams G. Isolation of a tumor factor responsible for angiogenesis. The Journal of Experimental Medicine, 1971;133(2):275-88.

8. Haraguchi, M, Watanabe A, Moriguchi S, Korenaga D, Maehara Y, Sugimachi $\mathrm{K}$. DNA ploidy is a major prognostic factor in advanced gastric carcinoma. 
Univariate and multivariate analysis. Surgery, 1991;110(5):814-9.

9. Ikeguchi M, Saito H, Maeta M, Oka S, Kondo A, Tsujitani S. Kaibara N. The expression of transforming growth factor-beta1 is significantly correlated with the expression of vascular endothelial growth factor and poor prognosis of patients with advanced gastric carcinoma. Cancer. 1999;86(8):1455-62.

10. Kimura K, Watanabe H. The diagnosis and treatment of early cancer. GUT, 1997;41(45):13E.

11. Korenaga D, Haraguchi M, Tsujitani S, Okamura T, Tamada R, Sugimachi $\mathrm{K}$, Inokuchi K. Cytophotometric DNA analysis of gastric carcinoma. Relationship between DNA ploidy and lymph node metastasis. Gan No Rinsho, 1986;32(1):45-9.

12. Korenaga D, Saito A, Baba H, Watanabe A, Okamura T, Maehara Y, Sugimachi K. Cytophotometrically determined DNA content, mitotic activity and lymph node metastasis in clinical gastric cancer. Surgery, St. Louis, 1990;107(3):2627.

13. Lima EB. Estudo citofotométrico do ADN de câncer gástrico. Curitiba, 2000. 80 f. Tese (Doutorado em Clínica Cirúrgica)- Setor de Ciências da Saúde, Universidade Federal do Paraná.
14. Ohyama S, Yonemura Y, Miyazaki I. Proliferative activity and malignancy in human gastric cancers. Significance of the proliferation rate and its clinical application. Cancer, 1992;69(2):314-21, 1992.

15. Rafaela Sierra. Cáncer gástrico, epidemiología y prevención. Acta méd. costarric. $2002 ; 44(2)$.

16. Sidransky D. Emerging molecular markers of cancer. Nat. Rev. Cancer. 2002;2(3):210-9.

17. Villaseca M, Araya JC, Roa I, Roa JC. Cáncer gástrico y regulación del crecimiento tumoral. Estudio de marcadores de proliferación celular y del complejo de proteinas. Rev. Med. Chil. 2000;128(2):127-36.

18. Yonemura Y, Miyazaki I. Quantitative estimation of malignancy of gastric cancer by DNA ploidy and proliferative activities. Rinsho Byori, 1990;38(9):10538 .

Conflito de interesse: não há Fonte financiadora: não há Recebido para publicação em: 06/05/2007 Aceito para publicação em: 30/06/2007 